\section{PIEZ01 gain-of-function mutations delay reticulocyte maturation in hereditary xerocytosis}

Dehydrated hereditary stomatocytosis 1 or hereditary xerocytosis (HX, OMIM 194380) is a rare hereditary autosomal dominant disorder characterized by hemolytic anemia and red blood cell (RBC) dehydration. The occurrence of HX is linked with gain-of-function mutations in PIEZO1, the gene encoding for the mechanosensitive non-specific cation channel PIEZO $1^{1,2}$ which is activated by shear-stress and in concert with other ion channels (particularly the Gardos potassium calcium-activated channel, KCNN4) regulates cell volume homeostasis and metabolic activity in the RBC. ${ }^{3}$ Intriguingly, PIEZO1 gainof-function mutations have recently been reported to occur at a much higher frequency within the population than had been previously described and have also been implicated in malaria resistance, ${ }^{4}$ suggesting that the mechanisms underpinning HX may merit further investigation. Since reticulocytosis is one of the hallmarks of $\mathrm{HX}^{5}$ we sought to determine whether altered reticulocyte maturation could be a causative agent of this phenotype. We characterize reticulocytes and erythrocytes from $10 \mathrm{HX}$ patients in comparison to healthy controls, revealing alterations in deformability and vesicle content that implicate a maturational defect in HX. We further demonstrate that HX patients suffer from impaired reticulocyte maturation as assayed through differences in the extent and rate of loss of CD71 and RNA content over time and that this effect can be recapitulated in healthy reticulocytes upon chemically-induced PIEZO1 overactivation, providing a functional link to the reticulocytosis phenotype present in HX.

A total of 10 samples were investigated in this study, constituting nine patients from six families with one sample in duplicate. The corresponding hematological parameters can be found in Table 1. All patients under investigation suffered from mutations in PIEZO1, with the majority displaying reticulocytosis and abnormal mean cell volume values (MCV). Several patients also displayed anomalous serum ferritin content, which comprised both iron overload and deficiency. Moreover, 3 of 9 patients had previously been subjected to splenectomy. Further detail on individual patients is provided in the Online Supplementary Patient Case Histories.

Splenectomy leads to a partial loss of the body's quality assurance system for ensuring the removal of physiologically-altered circulating $\mathrm{RBC},{ }^{6}$ the effects of which can be readily observed on the deformability index (DI) and cross-sectional area profiles obtained upon examination of the cells with the Automated Rheoscope and Cell Analyzer (ARCA), ${ }^{7}$ shown in Figure 1A. Individual scatter plots for each sample are shown in the Online Supplementary Figure S1.

Both $\mathrm{RBC}\left(\mathrm{CD} 71^{-}\right)$and reticulocytes $\left(\mathrm{CD} 71^{+}\right)$from splenectomized PIEZO1-defective patients display decreased deformability (CD71 median DI: 1.33 [Interquartile range (IOR): $1.08-1.58$ ], $\mathrm{CD} 71^{+}$median DI: 1.80 [IOR: 1.61-1.99]) in comparison with non-splenectomized patients (CD71 median DI: 1.70 [IOR: 1.461.94], $\mathrm{CD} 71^{+}$median DI: 1.91 [IOR: 1.76-2.06]), with their RBC displaying an enriched proportion of microcytic cells (Cross-sectional area $=\sim 40 \mu \mathrm{m}^{2}$ ). Interestingly, while RBC from non-splenectomized patients also display lowered deformability and macrocytosis in comparison with reference values (ref: CD71- median DI: 1.94 [IOR: 1.78-2.11]), their reticulocytes display a relatively normal DI profile (ref: $\mathrm{CD}^{+} 1^{+}$median DI: 1.93 [IOR: 1.84-2.02]). Whilst a potential influence of $C D 71$ reticulocytes on the total RBC population cannot be excluded, these results nonetheless illustrate that the effect of PIEZO1 overactivation on deformability only manifests in the transition from the reticulocyte to the $\mathrm{RBC}$ as defined by the loss of CD71, implicating a potential defect in reticulocyte maturation of HX patients.

Using mitochondrial content as a surrogate measure for intracellular vesicle content, as previously described, ${ }^{8}$ we proceeded to investigate whether physiological vesicle loss is altered in reticulocytes from HX patients, observ-

Table 1. Hematological parameters of the hereditary xerocytosis patients under investigation.

\begin{tabular}{|c|c|c|c|c|c|c|c|c|c|}
\hline Patient & Age & $\operatorname{Sex}$ & Mutation & Splenectomy & $\begin{array}{l}\text { Hemoglobin } \\
\text { (g/dL) }\end{array}$ & $\begin{array}{l}\text { MCV } \\
\text { (it) }\end{array}$ & $\begin{array}{l}\text { Reticulocyte no. } \\
\left(10^{\circ} / \mathbf{L}\right)\end{array}$ & $\begin{array}{l}\text { Reticulocyte } \\
\%\end{array}$ & $\begin{array}{l}\text { Ferritin } \\
(\mu g / L)\end{array}$ \\
\hline 1 & 33 & $\mathrm{M}$ & $\begin{array}{c}\text { p.R2088G + } \\
\text { p.2169-2170 delK }\end{array}$ & $\mathrm{N}$ & 14.2 & 105 & 718 & 18.7 & 94 \\
\hline $2^{+}$ & 23 & $\mathrm{~F}$ & p.R2456H & Y & 13.1 & 100 & 259 & 7.2 & 170 \\
\hline $3^{+}$ & 47 & M & p.R2456H & $\mathrm{N}$ & 16.8 & 93 & 997 & 20.0 & 107 \\
\hline 4 & 19 & M & p.V598M & Y & 16.1 & 106 & 472 & 11.0 & 591 \\
\hline $5^{\ddagger}$ & 47 & $\mathrm{~F}$ & p.E2496ELE & Y & 10.2 & 77 & 227 & 5.0 & 67 \\
\hline $6^{\ddagger}$ & 47 & $\mathrm{~F}$ & p.E2496ELE & Y & 12.5 & 74.4 & 113 & 2.6 & --.-- \\
\hline 7 & 55 & $\mathrm{~F}$ & p.V598M & $\mathrm{N}$ & 8.7 & 108 & 137 & 5.9 & 875 \\
\hline $8^{\sharp}$ & 46 & M & p.R2456H & $\mathrm{N}$ & 13.5 & 97 & 700 & 18.4 & 175 \\
\hline $9^{\sharp}$ & 43 & $\mathrm{~F}$ & p.R2456H & $\mathrm{N}$ & 11.8 & 94 & 294 & 9.3 & 257 \\
\hline $10^{+}$ & 18 & M & p.R2456H & $\mathrm{N}$ & 9.83 & 80 & 402 & 11.0 & 17 \\
\hline
\end{tabular}

The patient numbers in the table are used to identify the respective samples in the reticulocyte maturation experiment shown in Figure 2B-C. Patient age and sex are provided. "Mutation" identifies the PIEZO1 mutation(s) found in the patient."Splenectomy" defines whether the patient has or has not undergone splenectomy. MCV: mean cell volume (obtained through the use of a CELL-DYN Sapphire system or a Sysmex XN-9000 system); reticulocyte no.: absolute reticulocyte number; reticulocyte \%: reticulocyte percentage as a function of all erythroid cells in circulation. Serum hemoglobin concentration and serum ferritin concentration values are also provided. Reference values are provided under each of the numerical columns. $\dagger$ and \# indicate that the patients are relatives; $¥$ indicates a repeat admission to the clinic (no serum ferritin concentration values were obtained for the second visit). 
ing a marked increase in vesicle content in both $\mathrm{RBC}$ and reticulocytes from splenectomized HX patients (Figure 1B). Surprisingly, a significantly increased vesicle content is also observed in RBC from non-splenectomized patients but not in their reticulocytes, once again indicating that defects occur in $\mathrm{HX}$ during reticulocyte transition to the RBC.

To investigate whether an alteration exists in reticulocyte maturation in HX, reticulocytes were isolated from patients and healthy donors and incubated in IMDM (Online Supplementary Materials and Methods as previously described) $)^{9}$ at $37^{\circ} \mathrm{C} 5 \% \mathrm{CO}_{2}$ for seven days (168 hours), either alone or in co-culture by layering the reticulocytes onto MS-5 cells (murine stromal cell line). Transferrin receptor (CD71) expression and RNA content (as measured by thiazole orange [TO]) were then examined by flow cytometry. Given that reticulocyte maturation normally occurs over a period of 24-48 hours after release in circulation, ${ }^{10}$ the time frame of the experiment was selected in order to ensure that the effects induced by incubation became saturating, i.e. that the cells had reached their maximum possible progression through the maturational process during the experimental conditions used. Co-culture with MS-5 cells was utilized in this study due to previously reported positive effects regarding the capacity of MS- 5 cells, or of the microenvironment they generate, to facilitate reticulocyte maturation in vitro. ${ }^{11}$ The exact mechanism through which MS-5 co-culture induces partial reticulocyte maturation has not been reported yet; however, physical cell-cell interaction between MS-5 cells and reticulocytes could constitute one of the contributors to that mechanism. Importantly, since PIEZO1 channel activity (and thus mechanotransduction) is altered in HX patients, effects resulting from physical interaction could be disrupted.

Representative examples of the CD71/TO loss exhibited during maturation by healthy controls and HX patients are shown in Figure 2A, comprising culture both with and without the presence of MS-5 cells. Delayed reduction in the levels of both markers is evident in HX patients, and especially so at the 24- and 48-hour timepoints; however, we also observed that cells from HX patients start the maturational process with higher levels of both CD71 expression and RNA content, an observation that is consistent with the delayed erythroid differentiation recently reported in HX patients. ${ }^{12}$ Thus, the longer 168-hour timepoint is valuable from the perspective that a delay over the course of 48 hours or even longer would not disrupt the result achieved at the end of maturation. Progression through maturation also differs in both controls and patients depending on whether reticulocytes are co-cultured with MS-5 cells, with RNA being lost independently of co-culture and a more substantial reduction in CD71 content occurring in cells undergoing co-culture. Despite these differences, reticulocyte maturation is significantly delayed in HX patients irrespective of the method used for incubation (Figure 2BC). The percentage of cells negative for CD71 and RNA is significantly lower in HX patients after seven days of incubation in both conditions, illustrating not only the existence of a delay in maturation but also an inability of patient reticulocytes to undergo complete maturation in this system.

In order to investigate whether the observed effect was a direct consequence of PIEZO1 over-activation, we examined whether this maturational phenotype could be recapitulated by treating healthy reticulocytes with Yoda1 (a specific chemical activator of PIEZO1) ${ }^{13}$ over an extended period of time, with the added advantage that this method enables the experiment to be initiated from a standpoint of identical starting CD71/TO profiles. We observe a significant delay in maturation rate (Figure 2D) upon treatment with $5 \mu \mathrm{M}$ of Yoda1 which becomes less pronounced over time, likely due to compensatory mechanisms being engaged as a result of continuous PIEZO1 activation. The Yoda1 concentration in use was chosen
A

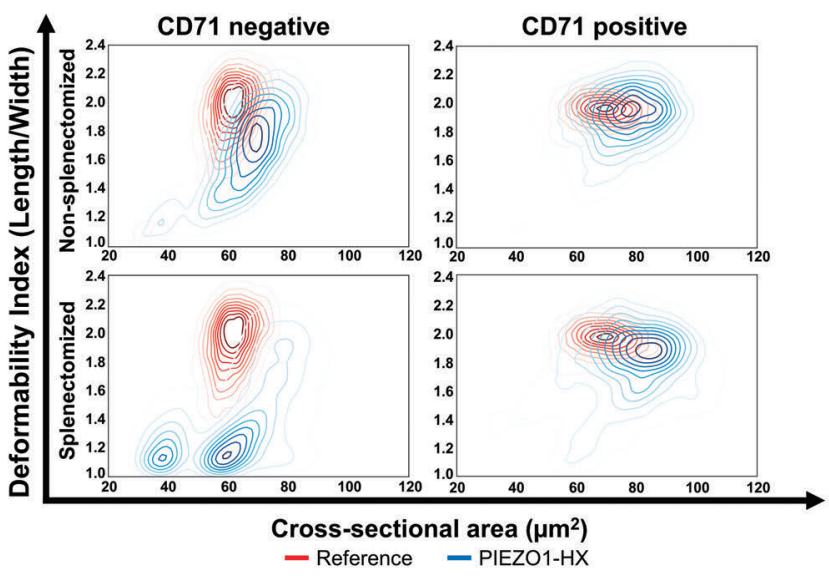

B

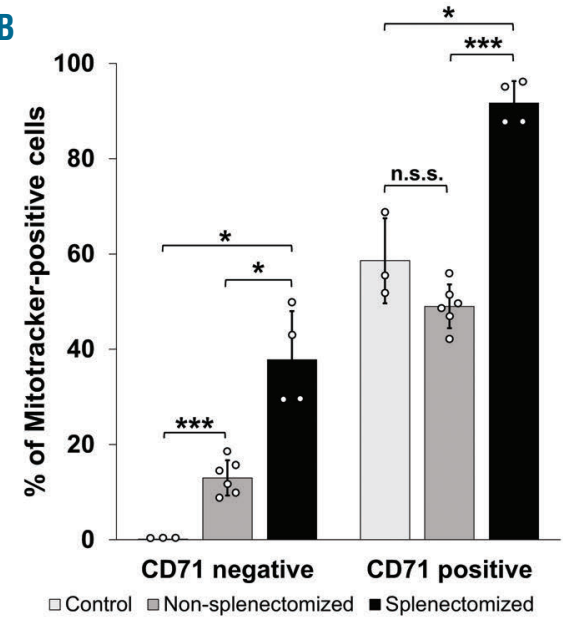

Figure 1. Cell defects arise upon reticulocyte maturation in hereditary xerocytosis patients. (A) Contour plots of cross-sectional area plotted against the deformability index (DI) (as measured by dividing cell length by cell width), visualizing the probability distribution of erythrocytes (CD71 negative) and reticulocytes (CD71 positive) from hereditary xerocytosis $(\mathrm{HX})$ patients compared to healthy reference samples and separated by splenectomy status. The cells were subjected to magnetic cell isolation using CD71 MicroBeads [Miltenyi Biotec] for separation of the CD71 positive and negative populations. The cells were analyzed with the Automated Rheoscope and Cell Analyzer, as previously described, ${ }^{8}$ with a minimum of 1,000 cells obtained per sample. The probability density functions were generated through kernel-density estimation of data comprising three reference samples, six non-splenectomized patient samples and four splenectomized patient samples. (B) Mitochondrial content in cells from HX patients compared to samples from healthy donors (control) in CD71 negative and CD71 positive cells, separated by splenectomy status. Tile scans composed of 10x10 images were taken at 1,024x1,024 resolution of cells labelled with Mitotracker Deep Red [500 nM, Thermo Fisher Scientific] using confocal imaging and analyzed manually. Sample numbers comprise three reference samples, six non-splenectomized patient samples and four splenectomized patient samples. Data are represented as mean \pm standard deviation (SD). All comparisons were made with two-sample unequal variance (heteroscedastic) t-tests. n.s.s.: non-statistically significant; $* P<0.05 ; * \star * P<0.001$. 
A

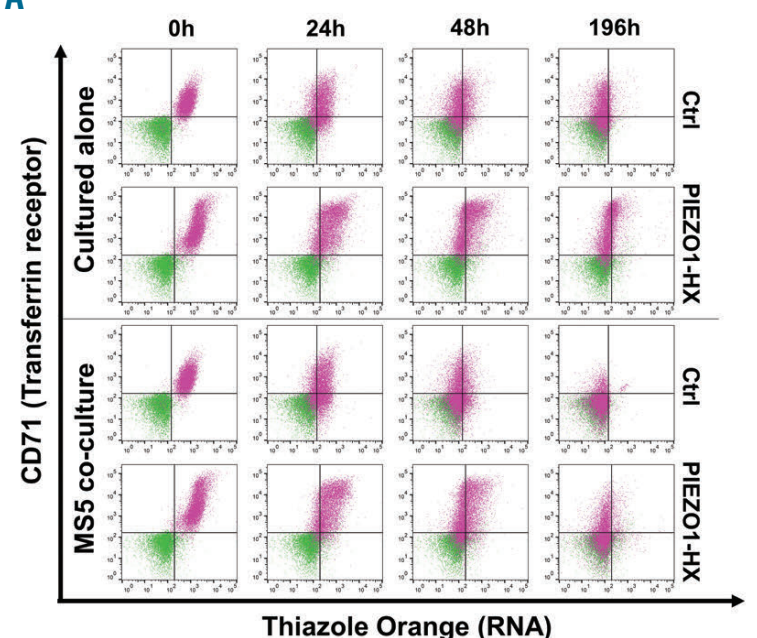

C

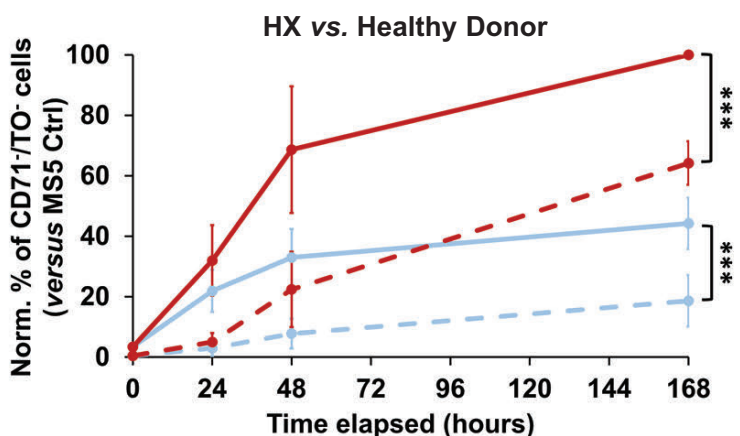

$\begin{array}{ll}\text { —.MS5 Ctrl } & \text { - No MS5 Ctrl } \\ \text {....MS5 PIEZO1-HX } & \text {.... No MS5 PIEZO1-HX }\end{array}$
B
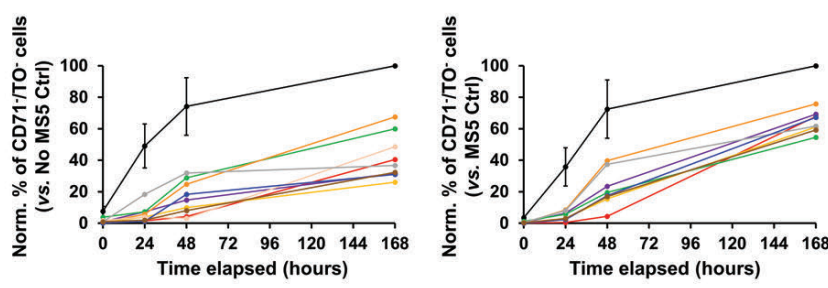

- Ctrl- $1-2-3-4-5-6-7-8-9$
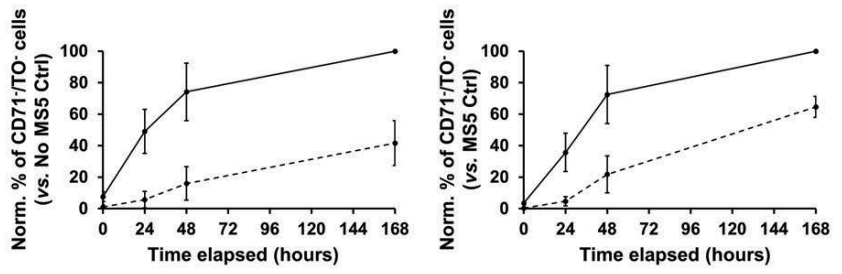

- Ctrl $\quad$... PIEZO1-HX

D

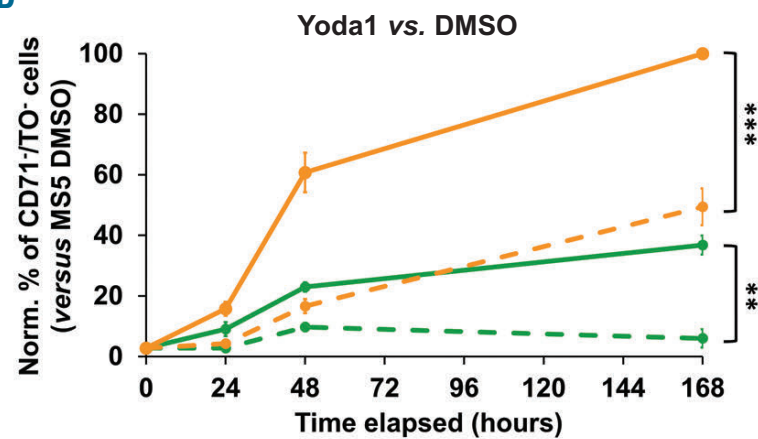

—.... MS5 DMSO MS5 Yoda1 $5 \mu \mathrm{M}$
. . MS

- No MS5 DMSO

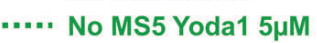

Figure 2. Delayed reticulocyte maturation is observed in hereditary xerocytosis and can be recapitulated through chemical activation of PIEZO1. (A) Representative flow cytometry diagram plotting RNA content (Thiazole Orange [TO]) against membrane transferrin receptor (CD71) content (APC anti-human CD71 [CY1G4], Biolegend), displaying progression through reticulocyte maturation of cells isolated from a healthy donor (Ctrl) or an hereditary xerocytosis (HX) patient (PIEZO1-HX, patient no. 5) and kept in static cell culture conditions over the course of 168 hours (seven days), shown in magenta. Cells were either cultured alone or layered onto MS-5 cells for co-culture (details for MS-5 culture are as described in Darghouth et al.)11 CD71-negative cells from the respective samples are shown in green, defining the quadrant and respective limits of a CD71-/TO- population. (B) Loss of CD71/TO in reticulocytes cultured alone (left) or in co-culture with MS-5 cells (right) as measured by the percentage of cells present in the CD71-/TO- quadrant and normalized against the CD71-/TO- percentage observed in the final timepoint of healthy control samples; $n=7$ for the control samples, with error bars showing standard deviation (SD). Each patient is represented as one separate line above, with all patients averaged below (the error bars show SD). In the bottom panel, all samples were normalized to the final timepoint of MS-5 co-cultured reticulocytes from the healthy control of their respective experiment. Co-culture data was not obtainable for patient 6 . The results from patient 10 were extreme outliers and thus excluded from this figure (available on request). Notably, this patient presented with iron deficiency, a condition which has previously been reported to decrease phenotypic severity in HX.15 (C) Average CD71/TO loss in reticulocytes from healthy donors and HX patients, cultured alone (blue) or in co-culture with MS-5 cells (red) and normalized against the CD71-/TO- percentage observed in the final timepoint of healthy control samples. Error bars show SD; $n=7$ for the control samples. All comparisons were made with two-sample unequal variance (heteroscedastic) $t$-tests. $* * * P$ value $<0.001$. (D) Average CD71/TO loss in reticulocytes cultured alone (green) or in co-culture with MS-5 cells (orange), with and without treatment with either 1:4,000 DMSO (v/v) or $5 \mu \mathrm{M}$ Yoda1 (chemical inducer of PIEZO1 activity; Tocris Bioscience). The data comprises three biological replicates, each with two technical replicates in MS-5 co-culture ( $\mathrm{n}=3$ for the non-MS5 data; $\mathrm{n}=6$ for the MS5 data). Error bars show SD. All comparisons were made with paired sample $t$ tests. $* * P<0.01 ; * * * P<0.001$.

due to constituting the maximal non-saturating concentration that induces calcium entry (Online Supplementary Figure S2). Notably, pharmacological treatment with FK506/Tacrolimus (a calcineurin inhibitor recently reported to abrogate Yoda1-induced effects in erythroblasts) ${ }^{12,14}$ did not ameliorate the delayed reticulocyte maturation phenotype of HX patients (Online Supplementary Figure S3).

Since disrupted calcium homeostasis is a prominent consequence of PIEZO1 overactivity, we hypothesize that elevated intracellular calcium levels account for the observed defects in reticulocyte maturation. However, as calcium is also known to exert widespread influence on cell signaling processes, determining the specific underlying mechanism for the detrimental effects caused by overactive PIEZO1 is a challenging proposition. Nonetheless, this work provides the first evidence that overactivation of PIEZO1 impacts reticulocyte maturation. Further investigation of the interplay between PIEZO1 activity and that of other ion channels (as well as of downstream signaling pathways) is likely to be of interest for future studies.

In conclusion, we report that hereditary xerocytosis patients with gain-of-function mutations in PIEZO1 suffer from multiple transcriptionally-independent effects caused by PIEZO1 overactivation. These include delayed 
reticulocyte maturation as assayed by loss of CD71, RNA and intracellular vesicle content and significantly decreased capacity to deform upon completion of maturation, which is exacerbated in patients that have undergone splenectomy. This delay in reticulocyte maturation can be recapitulated through chemical treatment with Yoda1, demonstrating that PIEZO1 overactivation has repercussions beyond impaired hydration and volume homeostasis in the erythrocyte.

\section{Pedro L. Moura, ${ }^{1,2}$ Bethan R. Hawley,}

Johannes G.G. Dobbe, ${ }^{4}$ Geert J. Streekstra, ${ }^{4}$

Minke A.E. Rab, 5,6 Paola Bianchi, Richard van Wijk,

Ashley M. Toye ${ }^{1,2,8 t}$ and Timothy J. Satchwell, ${ }^{1,2,8 \#}$

${ }^{*} A M T$ and TJS contributed equally as co-senior authors.

${ }^{1}$ School of Biochemistry, University of Bristol, Bristol, UK; ${ }^{2}$ NIHR Blood and Transplant Research Unit in Red Cell Products, University of Bristol, Bristol, UK; ${ }^{3}$ School of Physiology, Pharmacology and Neuroscience, University of Bristol,Britsol, UK; ${ }^{4}$ Amsterdam UMC, University of Amsterdam, Department of Biomedical Engineering and Physics, Amsterdam, the Netherlands; ${ }^{5}$ Department of Clinical Chemistry and Haematology, University Medical Center Utrecht, Utrecht University, Utrecht, the Netherlands; ${ }^{6}$ Van Creveldkliniek, University Medical Center Utrecht, Utrecht University, Utrecht, the Netherlands; "UOC Ematologia, UOS Fisiopatologia delle Anemie, Fondazione IRCCS Ca' Granda Ospedale Maggiore Policlinico, Milano, Italy and ${ }^{8}$ Bristol Institute for Transfusion Sciences, National Health Service Blood and Transplant (NHSBT), Bristol, UK

Correspondence:

TIMOTHY J.SATCHWELL - t.satchwell@bristol.ac.uk

ASHLEY M.TOYE - ash.m.toye@bristol.ac.uk

doi:10.3324/haematol.2019.231159

Acknowledgments: the authors would like to thank the donors, patients and their family members for their willingness to participate in this research. the authors thank the Wolfson Bioimaging Facility of the University of Bristol for use of their confocal systems, as well as the $M R C$ for establishing the Facility and the BBSRC Alert 13 capital grant (BB/L014181/1) for funding their acquisition of the Leica SP8.

Funding: PLM was funded by the European Union (H2020MSCA-ITN-2015, "RELEVANCE", Grant agreement number 675117). MR is supported by the Eurostars grant estar18105 and by an unrestricted grant provided by $R R$ Mechatronics. $P B$ was funded by the Fondazione IRCCS Ca' Granda Ospedale Maggiore Policlinico, Grant no. 2019 175/02, 2019. AMT and TJS were funded/supported by an NHS Blood and Transplant (NHSBT) RED grant (WP15-05) and the National Institute for Health Research Blood and Transfusion Research Unit (NIHR BTRU) in Red Cell Products (NIHR-BTRU-2015-10032). The views expressed are those of the author(s) and not necessarily those of the NIHR or the Department of Health and Social Care.
Information on authorship, contributions, and financial \& other disclosures was provided by the authors and is available with the online version of this article at www. haematologica.org.

\section{References}

1. Zarychanski R, Schulz VP, Houston BL, et al. Mutations in the mechanotransduction protein PIEZO1 are associated with hereditary xerocytosis. Blood. 2012;120(9):1908-1915.

2. Andolfo I, Alper SL, De Franceschi L, et al. Multiple clinical forms of dehydrated hereditary stomatocytosis arise from mutations in PIEZO1. Blood. 2013;121(19):3925-3935, S1-12.

3. Cahalan SM, Lukacs V, Ranade SS, Chien S, Bandell M, Patapoutian A. Piezo1 links mechanical forces to red blood cell volume. Elife. $2015 ; 4$

4. Ma S, Cahalan S, LaMonte G, et al. Common PIEZO1 allele in african populations causes RBC dehydration and attenuates plasmodium infection. Cell. 2018;173(2):443-455.

5. Andolfo I, Russo R, Rosato BE, et al. Genotype-phenotype correlation and risk stratification in a cohort of 123 hereditary stomatocytosis patients. Am J Hematol. 2018;93(12):1509-1517.

6. Pivkin IV, Peng Z, Karniadakis GE, Buffet PA, Dao M, Suresh S. Biomechanics of red blood cells in human spleen and consequences for physiology and disease. Proc Natl Acad Sci U S A. 2016;113(28):7804-7809.

7. Dobbe JG, Streekstra GJ, Hardeman MR, Ince C, Grimbergen CA. Measurement of the distribution of red blood cell deformability using an automated rheoscope. Cytometry. 2002;50(6):313-325.

8. Moura PL, Hawley BR, Mankelow TJ, et al. Non-muscle myosin II drives vesicle loss during human reticulocyte maturation. Haematologica. 2018;103(12):1997-2007.

9. Griffiths RE, Kupzig S, Cogan N, et al. Maturing reticulocytes internalize plasma membrane in glycophorin A-containing vesicles that fuse with autophagosomes before exocytosis. Blood. 2012;119(26): 6296-6306.

10. Johnstone RM, Adam M, Hammond JR, Orr L, Turbide C. Vesicle formation during reticulocyte maturation. Association of plasma membrane activities with released vesicles (exosomes). J Biol Chem. 1987;262(19):9412-9420.

11. Darghouth D, Giarratana MC, Oliveira L, et al. Bio-engineered and native red blood cells from cord blood exhibit the same metabolomic profile. Haematologica. 2016;101(6):e220-222.

12. Caulier A, Jankovsky N, Demont Y, et al. PIEZO1 activation delays erythroid differentiation of normal and Hereditary Xerocytosisderived human progenitors. Haematologica. 2020;105(3):610-622.

13. Syeda R, Xu J, Dubin AE, Coste B, et al. Chemical activation of the mechanotransduction channel Piezo1. Elife. 2015;4.

14. Aglialoro F, Yagci N, von Lindern M, van Wijk R, van den Akker E. A novel role for PIEZO1 in calcium homeostasis during erythropoiesis. Blood. 2018;132(Suppl 1):2321-2321.

15. Orvain C, Da Costa L, Van Wijk R, et al. Inherited or acquired modifiers of iron status may dramatically affect the phenotype in dehydrated hereditary stomatocytosis. Eur J Haematol. 2018;101(4):566569. 